\title{
Tachyon Mechanics and Classical Tunnel Effect.
}

H. K. WIMMEL

Max-Planck Institut für Plasmaphysik - Garching

(Lett. Nuovo Cimento, 2, 363 (1971))

Equation (7), first formula, should read $x^{\prime}=\Gamma(x+V t)$.

Equation (10) should read $\sum \tilde{p}^{\mu}=$ constant in time.

Equation (14), last formula, should read $W^{\mu}=Z \tilde{W}^{\mu}$. 